\title{
Chili Pepper Scent: Study and Recognition with Chemiresistors Array ${ }^{\dagger}$
}

\author{
Ettore Massera 1,*, Veronica Sberveglieri ${ }^{2}$, Estefania Núñez-Carmona ${ }^{3}$, Saverio De Vito ${ }^{1}$, \\ Vardan Galstyan ${ }^{3}$, Dario Zappa ${ }^{3}$, Maria Lucia Miglietta ${ }^{2}$, Brigida Alfano ${ }^{2}$ and \\ Girolamo Di Francia ${ }^{2}$ \\ 1 ENEA C.R. Portici P.le E. Fermi 1, Portici, I-80055 Naples, Italy; saverio.devito@enea.it \\ 2 CNR-IBBR Institute of Biosciences and Bioresources, Via Madonna del Piano, 10 Sesto Fiorentino, Firenze, \\ Italy; veronica.sberveglieri@ibbr.cnr.it (V.S.); mara.miglietta@enea.it (M.L.M.); \\ brigida.alfano@enea.it (B.A.); girolamo.difrancia@enea.it (G.D.F.) \\ 3 Department of Information Engineering, University of Brescia, Via Branze 48, Brescia, Italy; \\ e.nunezcarmona@unibs.it (E.N.-C.); vardan.galstyan@unibs.it (V.G.); dario.zappa@unibs.it (D.Z.) \\ * Correspondence: ettore.massera@enea.it; Tel.: +39-081-772-3388 \\ + Presented at the Eurosensors 2017 Conference, Paris, France, 3-6 September 2017. \\ Published: 15 August 2017
}

\begin{abstract}
Chili peppers are spices worldwide appreciated and represent a real culinary tradition and cultural identity for several populations. Here we present an effort to evaluate whether an array of chemiresistor gas sensors is capable to distinguish different chili pepper samples by the analysis of the emitted aroma. Our approach is based on two methods. A classical method based on GC-MSSPME to characterize intensity and composition of chemical compounds emitted by the fruits and a novel approach on Multivariate sensors response that produces an online graphic representation of the sensor features.
\end{abstract}

Keywords: scent; chemiresistor; e-nose; artificial nose; volatile organic compounds; GC-MS-SPME; food security; food safety

\section{Introduction}

Food authenticity and adulteration is a nowadays topic trend. Sensor Systems in food quality and security control assessment are strongly requested [1,2]. The wide range of chili peppers cultivars is a suitable and poor explored test [3] for embedded system sensors.

The most important parameter of food from the sensory point of view it's the aroma. It is composed by the set of volatile organic compounds (VOC) that form the so called fingerprint. The characterization of the volatile fingerprint may offer information about safety and quality and could be use as an indicator resource when it varies somehow from the standard. The set of VOC could provide also information about geographical origins and cultivars [4].

\section{Materials and Methods}

Chili peppers are fruit of plants from the genus Capsicum, members of the nightshade family, Solanaceae. Today in the world are cultivated five Species of Capsicum that have generated more than 50.000 cultivars. Measurements starts from six Capsicum cultivars belonging from three of the five major species of cultivated chili peppers: from the Species Annuum we test "tondo Calabrese" (TCCA) with a taste similar to sweet bell peppers and "Goat's Weed" (GWCA); from the Species Baccatum we test "Jamy" (JBC) often very sweet; from the Species Chinense we test "Trinidad Perfume" (TPCC), "Fatali", "Habanero Orange" and "Habanero red" very aromatic and often fruity, 
with a bitter, soapy aftertaste in some cultivars, mostly the superhot ones. This variability was needed so as to highlight the aromatic differences between both the species and the single cultivars [5].

\subsection{Analytical Method}

To extract and identify the set of VOCs fraction the pepper fruits were divided in parts placed in a $20 \mathrm{~mL}$ chromatographic vial. Vials were closed hermetically with silicon-PTFE septum, crimped with an aluminum crimp. To perform the HS-SPME analysis a triphasic fiber DVB/CAR/PDMS50/30 $\mu \mathrm{m}$ (Supelco Co. Bellefonte, PA, USA) and a HT280T auto-sampler (HTA S.r.l., Brescia, Italy). The vials were incubated at $50^{\circ} \mathrm{C}$ for 15 to reach the equilibrium, subsequently, the fiber was exposed at the same conditions. Desorption of volatiles took place in the injector of the GC-MS for 6 min at $250{ }^{\circ} \mathrm{C}$. For the analysis of the volatile set of peppers was used a Shimadzu Gas Chromatograph GC2010 PLUS (Kyoto, KYT, Japan) interfaced with a Shimadzu single quadrupole Mass Spectrometer MS-QP2010 Ultra (Kyoto, KYT, Japan). GC separations were performed on a DB-WAX capillary column $(30 \mathrm{~m} \times 0.25 \mathrm{~mm} \times 0.25 \mu \mathrm{m}$, Agilent Technologies, Santa Clara, CA, USA). Ultrapure helium $(99.99 \%)$ was used as the carrier gas at the constant flow rate of $1.0 \mathrm{~mL} / \mathrm{min}$. The GC oven temperature was programmed as follows: initially the column was held at $50^{\circ} \mathrm{C}$ for $1 \mathrm{~min}$ followed by a rise from 50 to $80{ }^{\circ} \mathrm{C}$ at $5{ }^{\circ} \mathrm{C} / \mathrm{min}$ and kept for $6 \mathrm{~min}$; finally a second rise from $80{ }^{\circ} \mathrm{C}$ to $250{ }^{\circ} \mathrm{C}$ at a rate of $7^{\circ} \mathrm{C} /$ min was performed and the temperature was maintained at $250^{\circ} \mathrm{C}$ for $1 \mathrm{~min}$. The identification of the volatile compounds was carried out using the NIST11 and the FFNSC2 libraries of mass spectra $[5,6]$.

\subsection{E-Nose Method}

Samples for the analysis are prepared cutting single fresh or unfreezed Chili pepper into pieces and placed in a 0.50cl PET bottle at room temperature. These samples are analyzed, within a few hours, sucking the air from the bottle by the mean of an HAPSITE H1552 Portable Gas Chromatograph with a Mass Spectroscopy detector (GC-MS). The same Sample is inhaled by the enose platform based on a max number of twelve chemiresistors [7]. Chemiresistor matrix can be divided in two typologies: 6 commercial heated sensors (TGS family) and 4 not heated nanostructured sensing films dropcasted onto transducer with an USB connector for an easily change on the sensor board (USB family). TGS sensors are FIGARO sensors of the 260, 261 and 262 families. USB sensors are affected by low dynamics, low stability and high humidity interference but, in spite of all, their low-temperature chemical kinetic can have a key role for odour discrimination.

Analysis is conducted without the use of a gas concentrator. For every Bottle-head-space of a cut chili pepper we produce a GC-MS chromatogram illustrating the principal chemical compounds emitted and a matrix 10 × 150 R_ij (Odour Pattern) based on 150 sampling of the ten sensors resistance $(j=1: 4$ USB sensors; $J=5: 10$ TGS sensors) measured every second during the e-nose analysis. Solid state sensors are affected by drift and it is a challenge to extract features useful for odour discrimination. We use the following steps to extract from the e-nose Odour Pattern parameters that can be used to compare different Odours:

- for the Sensor $\mathrm{j}$ baseline R0_j is calculated taking into account the mean value of 30 sampling before odour injection;

- $\quad$ percentage relative response: $\left.R s p \_j=\max \left|R \_i j-R 0 \_j\right|\right) / R 0 \_j * 100$;

- relative response velocity (signal differential with sampling at $1 \mathrm{~Hz})$ : Vrsp_ij $=\left(R_{-} i j-R_{-}(I-\right.$ 1)j)/R0_j ;

- $\quad \max$ relative response velocity: MVrsp_j $=\max \left|R_{-} i j-R_{-}(i-1) j\right| / R 0 \_j$. This parameter can be calculated both during the odour inhaling phase (ads) that during the odour exhaling phase (des);

- a new homogenized matrix S_ij $(20 \times 150)$ composed by $\left(R_{s p} \_j-R \_i j\right) / R s p \_j$ for $j=1: 10$ and Vrsp_ij/MVrsp_j for j = 11:20;

- Taking into account the USB Sensor that give the best MVrsp and the TGS Sensor that give the Best Mvrsp we calculate RMVrsp_j as the ratio of the MVrsp to the best one for each Sensor family. 
In this manner became possible to compare different Odour Pattern simply by subtracting their RMVrsp and their S_ij. We choose max relative response velocity instead of percentage relative response because we have reason to believe [8] that this differential parameter is less affected by sensors drifting, and saturation although it contains the same discrimination feature of the Sensor Response.

\section{Results and Discussion}

\subsection{Chili Pepper Scent Chemical Composition}

Analytical measurements performed through GC-MS-SPME describe a chemical pattern emitted by the chilli pepper samples composed by more than 160 chemical compounds. GC-MS data have been recorded for all the four different cultivars producing 12 chromatograms ( 3 for each cultivar). Data have been normalized and projected on the 3D principal component (PC) subspace. The patterns of the four cultivars appear to be significantly separated in the PC space supporting the occurrence of a specific olfactive fingerprint to be exploited by an e-nose. JCB data express a significant correlation with Caryophyllene, Acetoin, 1,6-Octadien-3-ol,3,7-dimethyl, GWCA and TCCA with Cyclopentane, 3-hexyl-1,1-dimethyl, Tetradecane,2-methyl-, 2,3-Butanediol, $\left[R-\left(R^{*}, R^{*}\right)\right]-$, finally TPCC cultivar is correlated with Hexyl n-valerate, Cyclohexanol,2,4-dimethyl-, Cyclohexanol, 3,3dimethyl-. Ethanol, 1-Butanol-3 methyl, Phosphonic acid (p-dydroxyphenil) and Phenylethyl Alcohol appear to be present in all the cultivar always eliciting a significant response with low intercultivar variance inducing a common mode signal that should be rejected by an e-nose. Apart from the above mentioned highly class-correlated analytes, a K-NN based wrapper analyte selection scheme (r-relief -Kira) [9] reported that Decanoicacid,ethyl ester, Oxacyclotetralon-2one-, 14 methyl and Citronellic Acid provides for the most discriminant information while 1-Butanol-3methyl, cis-2methyl-7-octadecene, Decanoic acid-silver provide a little amount of information in order to discriminate among the four different cultivar in a supervised scheme. Both unsupervised and supervised schemes agree that 1-Butanol-3methyl do not provide useful discriminant information.

\subsection{E-Nose Odour Comparison between Habanero and Fatalii}

These two cultivars were tested 3 times: first time with two fresh fruits, the other two time with six unfreezed fruits. GC-MS analysis shows the abundance of hexyl valerate and ethanol for all the samples. For the GC-MS habanero orange and red emitted similar chemical compounds while in Fatalii is not present the TrimethylDihydrofuran 2-1. E-nose odour pattern are acquired taking into account odour intensity and avoiding e-nose chamber contamination. Perhaps we use an odour dilution too high for the USB sensors causing a lack of stability in the USB odour Pattern.

In the all comparison TGS sensors shows minimal differences both in the histogram and in the comparison map (in Figure 1 an example of comparison). USB sensors seem to discriminate habanero from fatalii with the third sensor that show always strong differences in all the comparison between habanero and fatalii. Also in the comparison map is possible to isolate a distortion region (black circle in figure) correlated to the difference between fatalii and habanero that disappear in the comparison between two fatalii. However, the instability of the USB family can affect the reliability of such a result. 


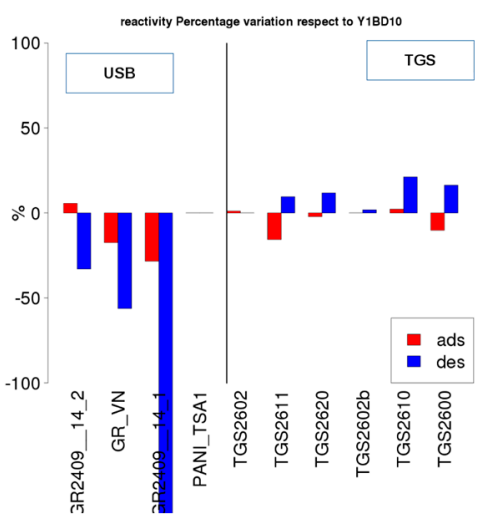

(a)

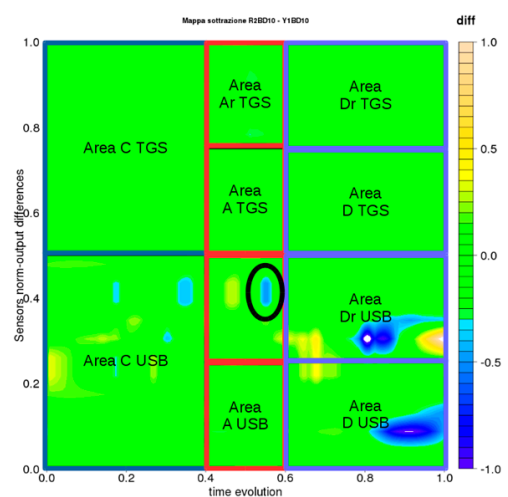

(b)

Figure 1. example of e-nose odor pattern comparison between Fatalii and Habanero for freezed samples: (a) percentage relative change in the sensors adsorbing and desorbing response max velocity; (b) Signal and Signal rate differences among sensors-comparison map. The black circle indicates an area that show discrimination capability.

\section{Conclusions}

This study try to answer to a question: if and how is possible to smell the chili peppers with an e-nose based on chemiresistive solid state sensors. We encountered several difficulties for the correct discrimination of cultivars with the e-nose: cultivar discrimination is based on second order variation of chemical compounds. So already the intensity variation of odours between the samples or e-nose chamber contamination can affect the discrimination reliability. Heated sensors TGS seems to go in crisis for cultivars discrimination so USB not heated sensing films became crucials if is possible to demonstrate enough reliability in the measurement. Work in progress deals with measuring a statistical substantial number of samples based on fresh fruits of different cultivars to confirm the enose cultivars discrimination capability.

Acknowledgments: The authors would like to thank the Association Pepperfriends (www.pepperfriends.com) for the support and the chili peppers supply.

Conflicts of Interest: The authors declare no conflict of interest.

\section{References}

1. Peris, M.; Escuder-Gilabert, L. Electronic noses and tongues to assess food authenticity and adulteration. Trends Food Sci. Technol. 2016, 58, 40-54.

2. Sberveglieri, V. Validation of Parmigiano Reggiano Cheese Aroma Authenticity, Categorized through the Use of an Array of Semiconductors Nanowire Device (S3). Materials 2016, 9, 81.

3. Gliszczyńska-Świgło, A.; Chmielewski, J. Electronic Nose as a Tool for Monitoring the Authenticity of Food. A Review. Food Anal. Methods 2017, 10, 1800-1806.

4. Sberveglieri, V.; Comini, E.; Zappa, D.; Pulvirenti, A.; Núñez Carmona, E. Electronic nose for the early detection of different types of indigenous mold contamination in green coffee. In Proceedings of the Seventh International Conference on Sensing Technology (ICST), Wellington, New Zealand, 3-5 December 2013; pp. 461-465.

5. Pruthi, J.S. Spices and Condiments; Chichester, E.M., Stewart, G.F., Eds.; Spices and Condiments; Academic Press: New York, NY, USA, 1980; p. 13.

6. Núñez Carmona, E.; Sberveglieri, V.; Ponzoni, A.; Zappa, D.; Pulvirenti, A. Detection of microbial contamination in potable water by Nanowire technology. In Proceedings of the 8th International Conference on Sensing Technology (ICST), Liverpool, UK, 2-4 September 2014; pp. 136-139.

7. Massera, E.; de Vito, S.; Formisano, F.; Buonanno, A.; Alfano, B.; di Francia, G.; Veneri, P.D. SNIFFI: A Portable development Gas Sensing embedded System. In Proceedings of the 2015 XVIII AISEM Annual Conference, Trento, Italy, 3-5 February 2015; pp. 1-4. 
8. Ricciardella, F., Massera, E., Polichetti, T., Miglietta, M.L.; Di Francia, G. A calibrated graphene-based chemi-sensor for sub parts-per-million NO2 detection operating at room temperature. Appl. Phys. Lett. 2014, $104,183502$.

9. Kira, K.; Rendell, L.A. A Practical Approach to Feature Selection. In Proceedings of the Ninth International Workshop on Machine Learning, 1992; pp. 249-256.

(C) 2017 by the authors. Licensee MDPI, Basel, Switzerland. This article is an open access article distributed under the terms and conditions of the Creative Commons Attribution (CC BY) license (http://creativecommons.org/licenses/by/4.0/). 